\title{
Advertisement-Aided Search in a P2P Context Distribution System
}

\author{
Irene Sygkouna, Miltiades Anagnostou, and Efstathios Sykas \\ Computer Networks Laboratory, School of Electrical and Computer Engineering, National \\ Technical University of Athens (NTUA), Greece \\ \{isygk, miltos, sykas\}atelecom.ntua.gr
}

\begin{abstract}
We study a $\mathrm{P} 2 \mathrm{P}$ proactive search mechanism based on the dissemination of advertisements for the new sources. The system design goal of limiting the state maintained by each peer and ensuring search efficiency is the driving reason for exploiting the hierarchical network model of the small-world idea. The results testify the theoretical bounds on search time and provide a view on the search time in relation to the directory capacity requirements of the peers.
\end{abstract}

\section{Introduction}

Context-aware services (CASs) need a flow of information from and about their environment in order to be able to adapt to it. Context producers, consumers and brokers are employed, with the last ones acting as mediatory players between producers and consumers. A consumer is a CAS that addresses context requests during its operation to the nearest broker. Producers are all the context sources. The need to support CASs that are highly robust and can scale well with the number of nodes and information sources points to Peer-to-Peer (P2P) architecture. The system faces the challenge to ensure efficient and scalable distribution of context information. Toward this direction we employ the advertisement dissemination mechanism, which implies that once a new source becomes available, the respective broker propagates an advertisement of the source to the remaining brokers. A broker may store a received advertisement in a local directory. Assuming infinite directory sizes, each peer broker stores each advertisement and thus maintains knowledge of all the sources available. However, as the system scales to a high number of sources, the length of the directories may become unbounded. Thus, our intention is to limit the directory capacity requirements of each peer and at the same time ensure low path-length for any search request. The idea is to make the peers store advertisements selectively, according to the philosophy of smallworld model. A requested object is then located in a bounded number of steps with a greedy search algorithm. We consider a set of brokers forming an overlay network. Each peer broker maintains the Local Sources Directory (LSD), with entries to the local sources, and the Remote Sources Directory (RSD), which caches directory entries for sources maintained by other peers. We assume that each source is described by a structure, the context object, that consists of a name and a set of attributes determined by (key, value) pairs. Search algorithms from literature are either request broadcastbased or advertisement-based. In the former case, the related mechanisms do not scale well with the network size since a search request is broadcast throughout the network, 
but improvements, such as Random Walk, have been proposed. Mechanisms of the latter case have been also proposed $[1,2]$ but have not addressed scalability problems.

\section{Search Mechanism with Advertisements}

Inspired by the hierarchical network model [3] of the small-world phenomenon we propose an advertisement dissemination mechanism, which proceeds in a way to make the information maintained in the RSDs reflect the links of the graph generated from the hierarchical model. The sources maintained by the overlay are classified according to a hierarchy, based on the names of the respective context objects. Thus, each source corresponds to a specific leaf of a complete $b$-ary context tree. Note that we allow more than one source to reside at a specific leaf since multiple sources may produce context objects of the same context name but different attributes.

Once a source enters the overlay through a node, an advertisement is disseminated to the rest of the peer nodes according to a flooding algorithm. A node stores a received advertisement if there is still room in its RSD and no other entry for a source belonging to the same leaf with the new source exists. Once the RSD becomes full, an elementary distribution of the entries is performed by corresponding each of the relevant remote sources to a local source in the following way: each remote source in turn is assigned to the nearest, in terms of tree distance, local source provided that this is feasible (does not exceed the threshold determined by the balanced distribution of the entries among the local sources). This way, a list of "concentrated" sources $L_{S_{i}}$ is created for each local source $S_{i}, i \in\left\{1,2, \ldots, L S D_{-} S I Z E\right\}$. Note that the distance between any two sources $X_{i}, X_{j}$, denoted by dist $\left(X_{i}, X_{j}\right)$, is measured by the height of the least common ancestor of the leaves hosting $X_{i}$ and $X_{j}$, respectively, in the tree. Note also that this procedure needs to be repeated every time a new source becomes available though a peer. When an advertisement of a new source is received by a node that would cause its RSD to exceed its size, the replacement scheme will take place. Assuming that the advertisement refers to a source $X$, a replacement can take place if the RSD does not contain any entry for a source that belongs to the same leaf with $X$. In particular, the following steps take place:

1. Among the local sources, the one that is closest to $X$ in terms of tree distance, say $S_{X}$, is selected, namely: $\operatorname{dist}\left(S_{X}, X\right)=\min _{S_{i} \in L S D} \operatorname{dist}\left(S_{i}, X\right)$.

2. From the respective list of concentrated sources $L_{S_{X}}$, a source that is furthest from $S_{X}$ in terms of tree distance, say $S_{X \_M A X}$ is selected: $\operatorname{dist}\left(\mathrm{S}_{\mathrm{X} \_\mathrm{MAX}}, S_{X}\right)=\max _{X_{i} \in L_{S_{X}}} \operatorname{dist}\left(X_{i}, S_{X}\right) . S_{X_{-} M A X}$ will then compete with $X$ for membership in the list. Based on the intuition from the model, $S_{X}$ should have a connection to $S_{X \_M A X}$ with probability proportional to $p_{1}=b^{-\operatorname{dist}\left(S_{X}, \mathrm{~S}_{\mathrm{X} \_\mathrm{MAXt}}\right)}$, and a connection to $X$ with probability proportional to $p_{2}=b^{-\operatorname{dist}\left(S_{X}, X\right)}$. 
According to the normalizing probabilities, $X$ will therefore replace $S_{X_{-} M A X}$ with probability equal to $\frac{p_{2}}{p_{1}+p_{2}}$.

Note that the advertisement of every new source that belongs to the same leaf with a local source is definitely stored, provided that the storage capacity is not exceeded.

The following greedy search algorithm is then applied: Once a peer receives a request and a matching source is not found in the LSD, it then searches its RSD. If a matching source is found, the request is forwarded to the peer pointed by the RSD. Otherwise, it looks up the nearest source (in terms of tree distance) in the RSD to the one requested, whose home-broker has not received the same request message from the current node in the past, in order to avoid cycles. It then forwards the request to the corresponding peer. The procedure is repeated until a matching source is located or the TTL is exceeded. Note that a step forward may incur a divergence from the target source if it cannot find in the RSD a source that is at least in the same distance from the target with the one selected during the previous step. Thus, two versions of the search algorithm are tested: the Advertisement Flooding-Forward (AF-F) makes only forward steps, while the Advertisement Flooding-Backwards (AF-B) makes a move backwards if it does not want to continue from its current node.

\section{Simulation Results}

We study and quantify the potential gain from the proposed mechanism with an event-based multithreaded simulation. As a reference for comparison we use Random, which applies for preprocessing a random replacement scheme at the RSDs, and for searching the same greedy algorithm. A number of sources join the overlay sequentially. After all the peer nodes have provided the same number of sources, a set of requests are initiated. We assume that the request popularity follows Zipf distribution [4] $(a=0.6)$. The performance of the mechanisms is well captured by the request hit ratio (the ratio of the number of successful requests to the total number of requests) and the average path-length per request (the ratio of the number of hops incurred across all requests to the total number of requests). If a request fails, it is deemed to have incurred TTL number of hops. Based on the hierarchical network model, the RSD_SIZE, not counting the entries for the sources that do belong to the same leaf with a local source, is computed as $R S D_{-} S I Z E=L S D_{-} S I Z E \cdot\left(c \cdot \log _{b}{ }^{2} n\right)$, with $n$ denoting the total number of leaves in the complete $b$-ary context tree and $c$ a constant. In effect, we will vary the RSD_size by varying the parameter $c$. Note that we set the LSD_SIZE to the size of the LSD just prior to executing the requests. We consider a $b$-ary tree with $b=4$ and height equal to 5 , for a total number of leaves equal to 1024. We also consider 200 peers. Setting the TTL to 20 and the LSD_SIZE initially to 10 (Fig. 1a) and then to 30 (Fig. 1b), we intend to explore how the performance metrics vary with respect to the RSD_SIZE. Parameter $c$ will vary from 0.2 to 1 with a step equal to 0.2 , changing the percentage of the RSD_SIZE to the total number of sources to $2.5 \%, 5 \%, 7.5 \%, 10 \%$, and $12.5 \%$, respectively. The metrics are calculated over 100 requests. The figure shows that the proposed mechanism clearly outperforms the Random, achieving high hit ratio and testifying the theoretical bound on search 
time $\left(O\left(\log _{b} n\right)\right)$. Obviously, as the value of $c$ increases, the performance improvement, compared to the Random, becomes less evident. Moreover, as the LSD_SIZE increases this performance divergence decreases more quickly with an increase in $c$. Thus, the value of the proposed algorithms is mainly identified under limited storage capacity of the RSD, since in this case the small-world connections are clearly shaped. Finally, the AF-F provides slightly better results compared to the AF-B.
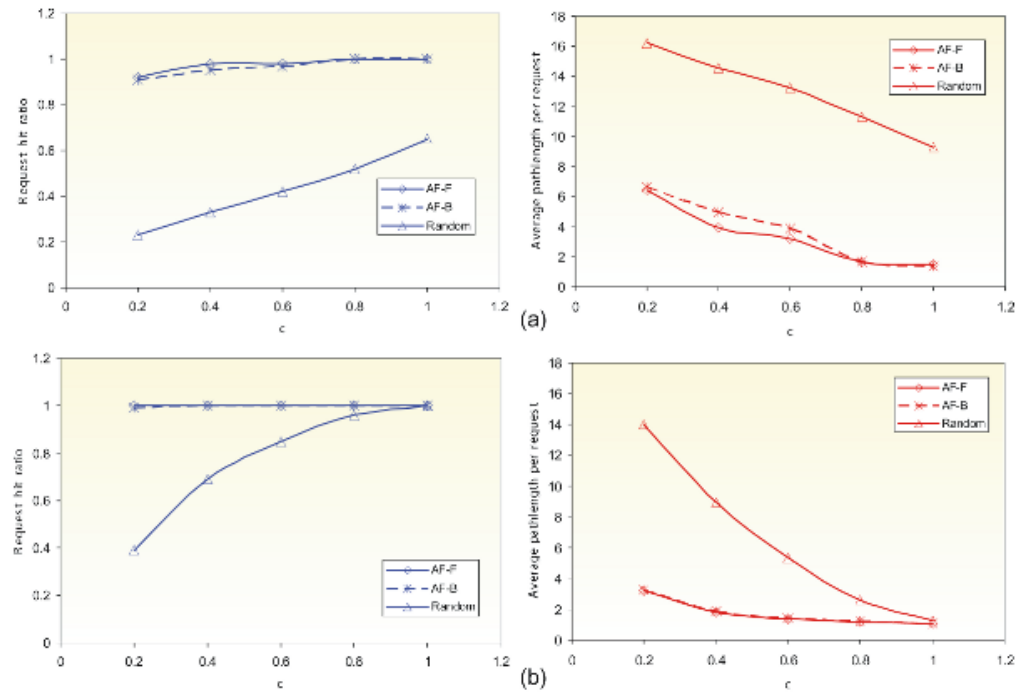

Fig. 1. Performance metrics after (a) 2000 and (b) 6000 sources have joined the system

\section{References}

1. A. Ranganathan and R.H. Campbell, "Advertising in a Pervasive Environment", Proceedings of the 2nd ACM International Workshop on Mobile Commerce, Atlanta, Georgia, pp.10-14, September 2002.

2. T. Finin, O. Ratsimor, A. Joshi and Y. Yesha, "eNcentive: A Framework for Intelligent Marketing in Mobile Peer-To-Peer Environments", Proceedings of the 5th International Conference on Electronic Commerce (ICEC'03), New York, NY, USA, pp.87-94, October 2003.

3. J. Kleinberg, "Small-world Phenomena and the Dynamics of Information", Advances in Neural Information Processing Systems (NIPS) 14, 2001.

4. L. Breslau, P. Cao, L. Fan, G. Phillips, and S. Shenker, "Web Caching and Zipf-like Distributions: Evidence and Implications", INFOCOM (1), pp.126-134, 1999. 